\title{
Patient noncompliance with therapeutic regimens and the factors of noncompliance in Gondar
}

\author{
Teferra Abula, Bpharm, Mpharm
}

\begin{abstract}
Noncompliance with drug treatment regimens is one of the many interacting factors which determine the outcome of therapy. This study was designed to emphasize the problem of non compliance with short-term medications. Five hundred ninety seven patients were randomly selected for a prospective study. Indirect methods such as patient interview and pill-count were employed for an assessment of compliance.

The overall compliance rate was $73.9 \%$, and the remaining $26.1 \%$ of the patients failed to comply with clinical prescriptions. Regardless of the status of compliance (compliant or noncompliant), an improvement of the illness was recorded in $73.2 \%$ of the patients. The remaining $26.8 \%$ of the patients had no change in the progress of their illnesses as subjectively assessed by the patients themselves. There is a significant association between the outcome of therapy and compliance $(p<0.01)$. The factors of noncompliance which have been reported by the noncompliant patients include non-redemption of drugs $(32.7 \%)$ forgetfulness $(18 \%)$, occurrence of adverse effects (16\%), misunderstanding of dosing instruction or lack of instruction (13.5\%), failure in the progress of the illness $(8.3 \%)$, improvement of the illness $(7.7 \%)$, and others $(3.8 \%)$. The importance of compliance from both the medical and the economical points of view and strategies towards improving compliance are discussed. [Ethiop. J. Health Dev. 2000;14(1):161
\end{abstract}

\section{Introduction}

Medication compliance has been defined in terms of an agreement between the patient's behavior of taking medications and the clinical prescription (1).

Full compliance of the patient with the physician's instructions can largely determine the successful outcome of therapy provided that a correct diagnosis and selection of medications are established.

Unfortunately, many patients, especially those who were prescribed medication for a long period of time were found not to comply with the physician's instructions (2-7) with a magnitude of the noncompliance rate ranging between $16.8 \%$ and $74.3 \%$ of the patients. InEthiopia, the defaulting rate for antituuberculosis drugs has been reported to be as high as $74.3 \%(2)$.

From the Department of Pharmacology, Gondar College of Medical Sciences, P.O. Box 196, Gondar, Ethiopia
The existing wide variation of the level of noncompliance in the literature may be due to the variability of the factors of noncompliance in different settings, the application of different methods for an assessment of compliance, and different views in the interpretation of compliance.

On the other hand, there are relatively few papers on compliance with short-term medications. The deterioration of compliance with time has also been described for shortterm medications (1). In another study about $7 \%$ of prescription forms issued in a mining general practice were not presented at pharmacies for dispensing (8), which indicates the noncompliance of the patients even to redeem their prescriptions. With regard to prescribed medicines, noncompliant behaviour includes major omissions, such as failure to cash prescription or to take any of the prescribed medicine, while lesser and probably more commonly encountered transgressions include errors in dosing, timing, and sequence 
of administration, and the taking of additional non-prescribed medicines $(1,9,10)$.

Noncompliance with drug treatment regimens is more likely to result in loss of treatment efficacy or overdose-related side effects although the consequences in the case of long-term medications may be of delayed onset.

To our knowledge, no study has addressed such an important public health issue in Ethiopia, particularly with short-term medications, where the problem of compliance is expected to be high.

Therefore, the main objective of the present study was to assess the level of compliance with medications prescribed for short periods of time and identify the factors of noncompliance in Gondar Town, North West Ethiopia.

The results of this study may contribute to develop strategies for improving the compliance of patients besides increasing the awareness of health care providers on the matter of compliance.

\section{Method}

This community-based, cross-sectional study was conducted during January-Júne 1998 to determine the magnitude and factors of noncompliance with medications prescribed for shot periods of time in Gondar Town, NorthWest Ethiopia.

The subjects of this study were out-patients who attended the out-patient departments of the Gondar Teaching Hospital and Gondar polyclinic (both located in Gondar Town with an estimated population of 120,000$)$.

The selected patients were dwellers of Gondar Town who have given their home addresses (easy-to-reach patients). Gondar Town was chosen because of its heavy population concentration in the Region which would facilitate the data collection process.

A simple random sample of 563 patients from the polyclinic and 34 patients from the hospital were selected and included in the study. All the patients were prescribed medications for short periods of time (a maximum of 10 days). The proportion of the patients from the hospital is small because most patients of the town prefer to go to the clinic close and takes less waiting time in the clinic unless their illness is severe. For this reason it was not possible to obtain equal sample sizes from the two medical care units during the study period.

Five hundred and ninety-seven patients were randomly selected and included in our study. Most of the patients (563) were from the clinic and only 34 from the hospital.

After obtaining an informed consent of the patients or other family members, patients were visited at their homes on the date they were expected to have finished the prescribed medication(s). Patients were not previously informed about the date of the visit nor the purpose of the visit previously. The purpose of the visit was explained by data collectors (three nurses and one pharmacist) on the date of the visit.

Indirect methods, such as patient interview and pill-count were employed for an assessment of compliance. The validity of these methods for an assessment of compliance was already described (11). After obtaining an informed consent, data on the usage of the medications (dosage, dosing interval, and duration of therapy), factor(s) of noncompliance, if any, and the outcome of therapy was obtained by patient interview. In addition, the source of dosing instructions and labelling information were inquired.

By the pill-count method, patients were asked to show their medication bottles and the remaining tablets, if any, or capsules were counted.

Patients who have taken their medication(s) as prescribed, were considered to be compliant, and those who did not take as prescribed were considered noncompliant. The definition of compliance in this study denotes $100 \%$ adherence to a clinical prescription. For example, a patient who missed even a single dose out of 28 prescribed doses was considered noncompliant. The remaining doses of analgesics/antipyretics were not used to imply noncompliance as these drugs were prescribed on "as needed" basis. 
The association between compliance and outcome of therapy was checed using the Chi Square Test.

\section{Results}

The mean age of the subjects was $25 \pm 16.2$ years and male/female ratio was 0.9 .

Intestinal parasitosis was the leading cause of morbidity $(21.3 \%$ ) followed by acute febrile illnesses $(10.6 \%)$, bronchitis $(9.3 \%)$, pneumonia $(7.5 \%)$, upper respiratory tract infection $(5.2 \%)$, and others (Table 1). The average diagnosis per patient was 1.1 . On the whole, 64 different types of drugs were prescribed out of which anti- infectives with a preponderance of antibacterials and analgesics/antipyretics were the most frequently prescribed individual drugs (Table 2). Out of 1186 prescription items prescribed as a whole, 1100 were oral medications, 72 injectables, and the remaining 14 were topical preparations. The number of drugs prescribed per patient ranged between 1 and 4 , the average number of drugs per patient being 1.98. The number of patients who were

Table 1: Commonly encountered diagnoses (frequency $=670)$, Gondar, 1998 .

\begin{tabular}{lcc}
\hline \multicolumn{1}{c}{ Diagnosis } & $\mathrm{n}$ & $(\%)$ \\
\hline 1. Intestinal parasitosis & 132 & $(21.3)$ \\
2. AFI & 71 & $(10.6)$ \\
3. Bronchitis & 62 & $(9.3)$ \\
4. Pneumonia & 50 & $(7.5)$ \\
5. URTI & 35 & $(5.2)$ \\
6. STD & 30 & $(4.5)$ \\
7. UTI & 25 & $(3.7)$ \\
8. Tonsilitis & 24 & $(3.6)$ \\
9. Malaria & 24 & $(3.6)$ \\
10. Gastritis & 23 & $(3.4)$ \\
11. Infected wound & 22 & $(3.3)$ \\
12. Diarrhoea & 17 & $(2.5)$ \\
13. Myalgia & 16 & $(2.4)$ \\
14. Asthma & 11 & $(1.6)$ \\
15. Others (50types) & 117 & $(17.5)$ \\
& &
\end{tabular}

AFI = Acute Febrile IIIness;

URTI = Upper Respiratory Tract Infection

STD = Sexually Transmitted Disease

$\mathrm{UT} \mid=$ Urinary Tract Infection
Table 2: Frequently prescribed individual drugs (frequency = 1186), Gondar, 1998 .

\begin{tabular}{lcc}
\multicolumn{1}{c}{ Drug } & $\mathrm{n}$ & $(\%)$ \\
\hline 1. Paracetamol & 162 & $(13.7)$ \\
2. Ampicillin & 113 & $(9.5)$ \\
3. Co-trimoxazole & 111 & $(9.4)$ \\
4. Acetyl salicylic acid & 105 & $(8.8)$ \\
5. Metronidazole & 78 & $(6.6)$ \\
6. Procaine penicilin & 55 & $(4.6)$ \\
7. Chloramphenicol & 51 & $(4.3)$ \\
8. Tetracycline & 49 & $(4.1)$ \\
9. Dipyrone & 40 & $(3.4)$ \\
10. Chloroquine & 38 & $(3.2)$ \\
11. Mebendazole & 38 & $(3.2)$ \\
12. Others (53 in kind) & 346 & $(29.2)$ \\
$\quad$ Total & 1186 & 100 \\
\hline
\end{tabular}

prescribed one drug, two drugs, three drugs, and four drugs was 155, 299, 129 and 14, respectively. The level of compliance and the outcome of therapy in both compliant and noncompliant groups is shown in Table 3. Four hundred and forty-one patients (73.9\%) were found to have taken their medications as prescribed (found to be compliant). The remaining $26.1 \%$ of the patients were found to be noncompliant. The percentage of patients who have reported an improvement of their illnesses was greater in the ompliant group than in the noncompliant group, and there exists a significant association between compliance and the positive outcome of therapy $(\mathrm{p}<0.01)$.

The compliance of patients was found to decrease as the number of prescribed drugs increases. Expectedly, the compliance with injectables was better than that of oral medication. The factors of noncompliance in 156(26.1\%) patients are shown in Table 4. Non-redemption of medication, forgetfulness and the occurrence of adverse effects were the most frequently reported factors of noncompliance. Most of the patients mentioned a single reason for noncompliance.

Lack of money and unavailability of the drug(s) in drug shops or pharmacies were the most frequently stated reasons for nonredemption of medications. 
Table 3: Compliance status ardo outcome of therapy $\{n=5971$, Gondar, 1998.

\begin{tabular}{|c|c|c|}
\hline Outcome of Therapy & Compliants $(n=441)$ & $\begin{array}{c}\text { Noncompliants }(n=156) \\
n \quad(\%)\end{array}$ \\
\hline Improvement & $(78.9)$ & (57.1) \\
\hline No change & $(20.1)$ & (42.9) \\
\hline
\end{tabular}

The level of the provision of information for patients with regard to their diseases and the prescribed drugs was recorded by asking the patients or caretakers whether they have been given such information. Prescriber's instructions about the aim of therapy and the dosing schedule was found to be low (27\%) whereas the provision of dosing instructions as well as labelling by dispensers was relatively high $(95 \%)$.

Table 4: Factors of noncompliance in $=156$ ). Gondar, 1998.

\begin{tabular}{lll}
\hline Factor & $n$ & $(\%)$ \\
& & \\
\hline Non-redemption of drug(s) & 51 & $(32.7)$ \\
Forgetfuness & 28 & $(18)$ \\
Occurrence of Adverse Effeci & 25 & $(16)$ \\
Misunderstanding or lack of instruction & 21 & $(13.5)$ \\
Failure in the progress of the disease & 13 & $(8.3)$ \\
Improvement of the disease & 12 & $(7.7)$ \\
Others * & 6 & $(3.8)$
\end{tabular}

* Other include: refusal to take, Muslim fasting, shift to traditional medicine, holiday drinks, death of relative

\section{Discussion}

The enormity of the problem of noncompliance even to medications prescribed for short periods of time, i.e., for acute illnesses, has been recognised in this study.

The fact that about $26 \%$ of the patients fail to adhere to clinical prescriptions and the existence of an association between compliance and the outcome of therapy deserves attention, particularly by health care providers. It is important to mention here that the outcome of therapy is the result of a set of interacting factors, such as socioeconomic, cultural, and others in addition to compliance with physicians' instructions for taking drugs. It is more likely that the magnitude of noncompliance is underestimated in this study because the methods usually overestimate compliance (11).

In the literature, it is pointed out that compliance with medications for acute illnesses deteriorates very rapidly, and the highest compliance rate for a 10 day oral penicillin course has been reported to be $89 \%$ (1), indicating a noncompliance rate of $11 \%$.

The comparison of our findings with other studies is somewhat difficult because of the following reasons.

Firstly, the application of different methods for an assessment of compliance and the existence of different views with regard to the definition of compliance may account for variations in the reported values.

Secondly, the potential factors of noncompliance can vary from country to country or even in different parts of the same country.

On the other hand, non-compliance rates for drugs prescribed for the treatment of chronic diseases, or for prophylactic purposes, are higher $(2-7,12)$, with wide variations of the reported values because of the above mentioned reasons.

Whether it is for long-term or for short-term medications, noncompliance decreases the maximum benefit that the patient may obtain from the therapeutic acumen although the consequences of non-compliance may be of a delayed onset in the former cases. Patient noncompliance is therefore expensive in terms of time, money, and other resources and can adversely affect the relationship between the patient and the physician.

The factors of non-compliance as a whole are related to the patient, the patient's illness, the physician, the drugs prescribed, and the treatment environment $(1,9,10)$.

The investigated factors of noncompliance in our study are also of the above mentioned 
origins.

Surprisingly, non-redemption of medication was the leading factor of noncompliance and the fact that $8.5 \%$ of the patients did not redeem $5.9 \%$ of the prescription items should draw the attention of all health care providers, especially physicians. Non-redemption duc to lack of money or unavailability of the drug in the pharmacy are beyond the capacity of the patients which should be considered by the health care delivery system inorder to maintain an adequate stock of the essential drugs and to allocate some free drugs for the extremely poor patients. Although non-redemption of prescription items has also been reported by other authors $(8,13)$, the fact that even very crucial drugs are not redeemed in our study may reflect a low socioeconomic standard of the study population.

Other factors of noncompliance resulted in minor omission of the prescription for which the patients or caretakers are largely responsible. Nevertheless, these factors could have been at least minimized had the patients been adequately instructed about their disease and the prescribed drugs.

The role of an adequate patient-therapist interaction for an improvement of compliance has been stressed as a key point for an intervention of the factors of noncompliance (14-16).

In this study, the provision of information about the disease, the aim of therapy, and the drugs by the prescribers to their patients was found to be low, only $27 \%$ of the patients receiving such information. The extension of the consultation time by some minutes for explanation of the diseases and the prescribed drugs should be considered to reinforce the participation of the patients in their treatment program.

A study conducted in Zimbabwe showed that $70 \%$ of the patients do not request any information from their physicians because of short consultation times (16). It is also not common in our communities that patients request information from the physicians.

Noncompliance with medication may well provide an alternative explanation for the discrepancy between prescribed medication and medical outcome (about $27 \%$ negative outcome in this study).

Therefore, educational strategies such as the provision of health education that includes the subject of compliance with prescribed medications by health educators and appropriate patient-therapist inceraction are recommended to improve the compliance of patients with medications.

The potential problems of poor compliance need larger scale studies to show if and how behavior can be altered to improve compliance.

\section{Acknowledgennent}

I would like to acknowledge the Ethiopian Science and Technology Commission for its financial support.

I am also indebted to Dr. Alemayehu Worku for his assistance in statistical analysis, to Ato Demeke Dessu for his valuable help in editing the manuscript, and to Wro. Lemlem Beyene for her technical assistance.

\section{References}

1. Sackett DL. The magnitude of compliance and noncompliance. In :Sackett DL and Haynes RB, eds. Compliance with therapeutic regimens. Baltimore, John Hopkins University Press, 1976:9-25.

2. Argaw $H$. Defaulting from anti-TB treatment in Jimma hospital, South West Ethiopia. Abstract: Sixth Annual Conference of EPHA, 6-8 Dec., 1995, Addis Ababa.

3. Dekker FW, Dieleman FE, Kaptein AA, Mulder JD. Compliance with pulmonary medication in general practice. Eur Respir $J$ 1993;6:886 890 .

4. Dowse $R$ and Futter WT. Outpatient compliance with theophylline and phenytoin therapy. SAMJ 1991;80:550-553.

5. Nageotte $C$, Sullivan $G$, Duan $\mathbb{N}$ and Camp PL. Medication Compliance among the seriously mentally ill in a public mental health stystem. Soc Psychiatr Epidemiol 1997;32:4956.

6. Tettersell MJ. Asthma patient's knowledge in relation to compliance with drug therapy. 
Journal of advanced Nursing. 1993;18:103113.

7. Wardman AG, Knox AJ, Muers MF and page RL. Profiles of noncompliance with antituberculosis Therapy. $\mathrm{Br} \mathrm{J}$ Dis, Chest 1988;82:285-289.

8. Waters WHR, Gould NV, Lunn JE. Undispensed prescriptions in a mining practice. BMJ 1976;1:1062-63.

9. Benet LZ.Principles of prescription order writing and patient compliance instructions In: Hardman JG, Limbird LE eds. The pharmacological basis of therapeutics, $g^{\text {th }}$ edition. New York :McGraw Hill press, 1996:1697-1706.

10. Griffith, S. A review of the factors associated with patient compliance and the taking of prescribed medicines. British Journal of general practice 1990;40:114-116.

11. Gordist L.Methodologic issues in the measurement of patient compliance. In: Sackett DL and Haynes RB, eds. Compliance with therapeutic regimens. Baltimore, John Hopkins University press, 1976:51-66.

12. Taylor P,Mutambu SL. Compliance with malaria chemoprophylaxis programmes in Zimbabwe. Acta Trop 1987;44:423-431.

13. Beardon PHG, McGilchrist MM, Mckendrick AD, McDevitt DG, MacDonald TM. Primary noncompliance with prescribed medication in primary care. BMJ 1993;307: 846-848.

14. Urquhart J. patient noncompliance with drug regimens: measurement, clinical correlates, economic impact. European Heart Journal 1996;17(supplement A): 8-15.

15. O'Brien MK, Petric K, Racburn J. Adherence to medication regimens: updating a complex medical issue. Medical care Review 1992;49:435-454.

16. Nyazema NZ, Chavunduka D, Dzimwasha $\mathrm{M}$, et al. Drug information for the community: type and source. Cent Afr J Med 1991;37:203206. 